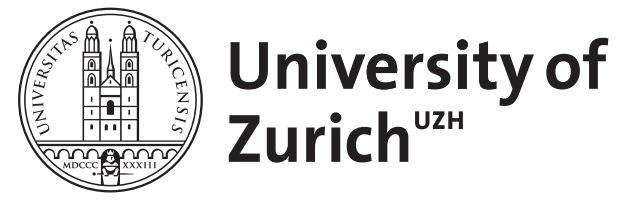

Zurich Open Repository and Archive

University of Zurich

University Library

Strickhofstrasse 39

CH-8057 Zurich

www.zora.uzh.ch

Year: 2012

\title{
Metaphysik der Emotionen
}

Renz, Ursula

Posted at the Zurich Open Repository and Archive, University of Zurich

ZORA URL: https://doi.org/10.5167/uzh-70192

Journal Article

Published Version

Originally published at:

Renz, Ursula (2012). Metaphysik der Emotionen. Deutsche Zeitschrift für Philosophie, 60(1):144-148. 


\title{
Metaphysik der Emotionen
}

\author{
Von URSULA RENZ (Klagenfurt)
}

DOMINIK PERLER: TRANSFORMATIONEN DER GEFÜHLE. Philosophische Emotionstheorien 1270-1670. S. Fischer Verlag, Frankfurt/M. 2011, 533 S.

Nachdem in der Philosophie der Gefühle während eines halben Jahrhunderts über die Natur, die kognitiven Leistungen und den moralischen Wert von Gefühlen diskutiert wurde, ist seit einigen Jahren auch ein erhöhtes Interesse an klassischen philosophischen Emotionstheorien zu beobachten. Mit seinem Buch Transformationen der Gefühle stellt sich Dominik Perler die Aufgabe, dieses Interesse einer breiteren Leserschaft zu vermitteln. Das ist ihm - soweit das Gelingen von Vermittlungsprozessen überhaupt von Autoren abhängig ist - in hervorragender Weise gelungen: Das Buch ist übersichtlich konzipiert, sehr klar und leichtfüßig geschrieben und zudem mit zahlreichen eingängigen Beispielen versehen, sodass selbst Ansätze, die auch für Philosophen eher schwer zugänglich sind, leicht verständlich werden. Und dies alles, ohne dass die historische Differenz zum Verschwinden gebracht würde. Im Gegenteil, indem Perler die jeweiligen Emotionstheorien stets im Lichte ihrer - zunächst oft abstrus anmutenden metaphysischen Voraussetzungen erörtert, führt er seine Leser in fremde Denkwelten ein, die sich weder einfach von unserem Alltagsverständnis her erschließen noch schlicht in eine Reihe mit gegenwärtigen (populär-)wissenschaftlichen Ansätzen gestellt werden können.

Nun ist solches nicht zu leisten, ohne dass im Blick auf die Auswahl und Anordnung des Stoffes einige prinzipielle Entscheidungen getroffen werden. So rollt Perler die Gefühlstheorien von Thomas von Aquin, Duns Scotus, Wilhelm von Ockham, Montaigne, Descartes und Spinoza im Wesentlichen entlang zweier thematischer Achsen auf. Die erste dieser Achsen bildet der bereits erwähnte Zusammenhang von Metaphysik und Gefühlstheorie oder genauer: die historische Transformation dieses Zusammenhangs. Dieser Fokus auf dem Zusammenhang von Metaphysik und Gefühlstheorie ist für die Anlage von Perlers Untersuchung in mehrfacher Hinsicht von Belang. Zum einen dürfte dies bei der Auswahl der vorgestellten Bezugsautoren eine nicht unwichtige Rolle gespielt haben. Es ist frappant, dass mit einer Ausnahme Philosophen berücksichtigt werden, die sowohl ausgeprägte metaphysische als auch gefühltheoretische Interessen hatten. Und auch die Ausnahme - Michel de Montaigne - passt quasi ex negativo zu dieser Linie: Wenn Montaigne, wie Perler zeigt (vgl. vor allem 209), die Methode der pyrrhonischen Skepsis auf emotionstheoretische Dogmen anwendet, dann wird auch die Annahme eines solchen Zusammenhangs relativiert. 
Zum anderen motiviert der Ansatz beim Zusammenhang von Metaphysik und Emotionstheorie auch Perlers Perspektive auf die historischen Veränderungen der behandelten Periode. Er versteht diese Veränderungen maßgeblich von der Ablösung der Naturphilosophie von der hylemorphistischen Metaphysik her. Dabei warnt er mit Recht davor, diese Ablösung als eine wissenschaftliche Revolution zu begreifen. Es handle sich eher um einen „Prozess der schleichenden Veränderung und Neuinterpretation“" (35), weswegen weder die Theorien vor 1600 noch jene danach als „,monolithischer Block“ wahrgenommen werden dürften (35). Gleichwohl begreift er diese Ablösung als einen „tiefgreifenden Umbruch“ (22), dessen Einfluss bis heute anhalte und dessen Erforschung daher auch für die gegenwärtige Debatte von Interesse sei. Und in der Tat: Wie Perler zu Recht geltend macht, ist Descartes' methodischer Ansatz bei „einer umfassenden Theorie über körperliche Reizaufnahme und kognitive Reizverarbeitung“ noch immer attraktiv (ebd.). Auch wenn uns viele Erklärungen Descartes' heute befremden, sind wir in methodischer Hinsicht noch immer Kartesianer.

Nicht weniger aufschlussreich ist die zweite thematische Achse in Perlers Darstellung, die mit der Frage zu tun hat, ob - und wenn ja wodurch - Emotionen transformiert oder mindestens reguliert werden können. Hintergrund dieser Frage ist die Tatsache, dass wir in unseren Alltagsüberlegungen von scheinbar widersprüchlichen Überzeugungen bezüglich der Beherrschbarkeit von Emotionen ausgehen. Auf der einen Seite haben wir die Intuition, dass wir für unsere Gefühle ,nichts können“, denn sie „passieren“ uns mehr oder weniger unwillkürlich. Auf der anderen Seite haben wir aber auch den Eindruck, unsere Gefühle irgendwie kontrollieren zu können: Wir „reißen uns zusammen“ und versuchen so unsere momentane Niedergedrücktheit zu überwinden. Die Frage stellt sich, ob diese beiden Intuitionen miteinander versöhnt werden können.

Wie Perler zeigt, beantworten sämtliche seiner Bezugsautoren diese Fragen insofern positiv, als sie eine indirekte Einflussnahme des Subjekts auf die eigenen Emotionen annehmen. Wir können zwar - so der gemeinsame Tenor seiner Rekonstruktionen von Thomas', Duns Scotus', Wilhelm von Ockhams, Montaignes, Descartes' und Spinozas Positionen - Emotionen nicht einfach per Knopfdruck an- oder abknipsen, aber wir können die mentalen Dispositionen verändern, die unseren Emotionen zu Grunde liegen. Keine Einigkeit besteht indes darüber, wie und warum eine solche indirekte Einflussnahme auf die eigenen Emotionen möglich ist. Damit verbunden besteht auch ein Dissens darüber, wie weit unser Einfluss reicht. Während beispielsweise Thomas den Akzent auf die rationale, durch den Intellekt gesteuerte Veränderung der unseren Emotionen zu Grunde liegenden Wahrnehmungen und Vorstellungen legt (105 f.), ist für den voluntaristischen Ansatz Ockhams vielmehr die willentliche Kontrolle der Aufmerksamkeit von Belang (189). Nochmals ein ganz anderes Modell treffen wir bei Montaigne an, für den jener Perspektivenwechsel entscheidend ist, den wir vollziehen, wenn wir die Sichtweise oder das Leiden anderer verstehen wollen (vgl. vor allem 273 sowie 267).

Was die Gründe und Reichweite der Möglichkeit einer reflexiven Einflussnahme auf die eigenen Emotionen betrifft, so wird nach Perler einmal mehr der Zusammenhang von Metaphysik und Gefühlstheorie wichtig, und zwar kommen hier nicht nur Fragen der Architektur der Seele, sondern auch kausal- und modaltheoretische Erwägungen zum Zuge. Wenn etwa Descartes trotz seiner mechanistischen Naturphilosophie relativ große Spielräume für die Beeinflussung der eigenen Emotionen zulässt, so liegt das wesentlich daran, dass er die Annahme einer absolut notwendigen Korrelation zwischen physiologischen und mentalen Zuständen zurückweist (vgl. vor allem 302 und 340). Umgekehrt sind bei Spinoza der Transformation von Emotionen dadurch Grenzen gesetzt, dass er nach Perlers Interpretation einen strengen Nezessitarismus vertritt. Demnach seien unsere mentalen Zustände nicht nur durch die kausale Vorgeschichte determiniert, sondern es gebe überdies „,nur eine mögliche Vorge- 
schichte“, weswegen immer schon und vollständig festgelegt sei, ,welcher Zustand zu einem bestimmten Zeitpunkt" auftrete (375).

Es ist klar: Vor diesem Hintergrund sind natürlich die Möglichkeiten einer Einflussnahme auf die eigenen Emotionen beschränkt. Perler zufolge beschränkt sich der Effekt von Spinozas rationaler Therapie denn auch darauf, dass wir eine vollständige Erkenntnis der eigenen Emotionen inklusive ihrer Ursachen haben. Eine solche Erkenntnis ist bei Spinoza zwar von einem Epiphänomen der Freude begleitet. Gleichwohl ist das natürlich ein Therapiekonzept, das unseren lebensweltlichen Erwartungen an eine Therapie nicht gerecht wird. Wer daher mehr von einer Therapie erwarte als jene bei Spinoza mit Freude gleichgesetzte vollständige Repräsentation, der müsse, so Perler, ,unweigerlich den gesamten metaphysischen Rahmen transformieren“ (442).

Man kann daher, wie Perler zu Recht geltend macht, auch mit Spinoza nicht beides haben, einen strikten, ausnahmslos geltenden metaphysischen Nezessitarismus und ein Therapiekonzept, das Alternativen und mithin metaphysische Möglichkeit voraussetzt. Meines Erachtens unterschätzt Perler hier allerdings, dass man im Blick auf diesen Punkt nicht nur als Gefühlstheoretiker, sondern auch als Spinoza-Exeget eine Entscheidung treffen muss. So ist Spinozas Modaltheorie von Inkonsistenzen geprägt, auf die unter anderen Hans-Peter Schütt vor längerem hingewiesen hat. ${ }^{1}$ Angesichts solcher Inkonsistenzen stellen sich Fragen, die mit einer Rekonstruktion von Spinozas Thesen und Argumenten allein nicht beantwortet werden können. So fragt sich zum einen, welche konkreten - etwa theologiegeschichtlichen oder politischen - Interessen und Problemlagen hinter solchen Inkonsistenzen stehen. Zum andern muss gerade der an der praktischen und politischen Philosophie interessierte Leser Spinozas eine Entscheidung treffen, was er lieber preisgeben will: die Annahme, dass Spinoza tatsächlich einem so strikten Nezessitarismus verpflichtet war, oder die zahlreichen Aussagen seiner Freiheitslehre, die gewisse Spielräume voraussetzen.

Einen eigentlichen Kontrapunkt zur gegenwärtigen Diskussion markiert das Kapitel zu Duns Scotus und Ockham. In der Gegenwartsdiskussion werden Gefühle vorrangig unter dem Gesichtspunkt ihrer Beziehung zu unseren Kognitionen thematisiert, wobei vor allem darüber gestritten wird, ob Emotionen eher Urteilen oder Wahrnehmungen gleichen. Ein anders gelagerter Dissens zeichnete sich im 13. und 14. Jahrhundert ab, wenn gefragt wurde, ob alle unsere Gefühle als sinnliche Regungen anzusehen sind oder ob man darüber hinaus nicht auch noch eine weitere Klasse von Emotionen annehmen muss, so genannte ,passiones' des Willens oder voluntative Emotionen. Bemerkenswert an diesem Begriff der voluntativen Emotion ist nicht allein, dass in ihm zwei scheinbar gegenläufige Annahmen verbunden werden: nämlich einerseits die bisweilen unter dem Stichwort der Urteilstheorie verhandelte These, dass in Gefühlen unsere Einstellungen gegenüber Werten oder allgemeiner gegenüber der Wert- und Sinnhaftigkeit der Welt zum Ausdruck kommen, andererseits die Überzeugung, dass selbst diese mit Werturteilen verbundenen Emotionen den Charakter von Widerfahrnissen haben. Darüber hinaus ist die Annahme einer zusätzlichen und weitaus rationaler gefassten Art von Emotionen vor allem auch deshalb von Belang, weil sie es eher als der, ,rationalistische Optimismus" von Thomas erlaubt, auch die Widersprüchlichkeiten, Ambivalenzen oder Schwankungen unseres emotionalen Lebens zu erklären.

Perler führt dies sehr schön anhand des bei Scotus diskutierten Beispiels der Emotionen Christi am Kreuz vor. Als Mensch, der er (auch) war, empfand Christus am Kreuz naturgemäß große Schmerzen, er war ihnen ausgeliefert wie jeder andere auch. Darüber hinaus hatte er vermutlich aber auch höherstufige Emotionen, wie etwa die Trauer über den Verrat oder die

1 H.-P. Schütt, Spinozas Theorie der Modalitäten, in: Neue Hefte für Philosophie, 24-25 (1985), 165183. 
Freude über die mit seinem Leiden verbundene Erlösung. Wie Perler deutlich macht, sind solche höherstufigen Emotionen für Scotus nicht einfach als Zustimmung zu Werturteilen zu verstehen, denn „Urteile haben an sich keine motivationale Kraft“, sondern nur als volitive Einstellung: „Nur wenn ich will, dass etwas nicht eintritt, und dieses Ereignis doch eintritt, werde ich traurig." (144) Wie Perler zeigt, macht es diese Vorgabe unter anderem möglich, emotionale Konflikte als eine Art „Widerstreit zwischen ,niederer" und ,höherer" Emotion“ (152) zu analysieren. Damit wird eine Dimension des emotionalen Lebens eingeholt, die in vielen Emotionstheorien unterbeleuchtet bleibt. Emotionale Konflikte sind oft nicht einfach, wie im Fall von Thomas' Beispiel des Schafs im Wolfspelz, das Perler mehrfach heranzieht, auf widersprüchliche Wahrnehmungen zurückzuführen. Sie können auch der Ausdruck eines tiefer greifenden seelischen Widerstreits sein.

Insgesamt ist also die Lektüre von Perlers Monographie auch für spezialisierte Leserinnen und Leser ein großer Gewinn. Dass dabei deren Bedürfnisse nicht allesamt befriedigt werden können und man den einen oder anderen Punkt gerne noch etwas genauer diskutieren würde, versteht sich von selbst. Auf zwei dieser Punkte möchte ich abschließend doch noch kurz hinweisen.

Mit seinem Fokus auf dem Zusammenhang von Metaphysik und Gefühlstheorie ist Perlers Darstellung von vorneherein auf Relativierung angelegt. Stellenweise wünschte man sich allerdings, dass die Diskussion über den Erkenntnisanspruch von Emotionstheorien stärker auf die Spitze getrieben würde. Selbst wenn man die Auffassung Perlers teilt, dass die konzeptuelle Ausbuchstabierung von Gefühlen stets von bestimmten metaphysischen Rahmenbedingungen abhängt, ist damit noch vieles offen. Perler selber zieht folgende Konsequenz: „Hat man erkannt, wie sehr dieser Rahmen von fundamentalen metaphysischen und methodologischen Annahmen abhängt und wie veränderbar diese Annahmen sind, wird man auch auf die historische Bedingtheit heutiger Erklärungsrahmen aufmerksam.“ (445) Dieser Hinweis auf die historische Bedingtheit von Emotionstheorien ist zweifelsohne richtig und wichtig. Doch betrifft die Einsicht in die historische Bedingtheit nur die Ebene der metaphysischen Voraussetzungen von Theorien? Oder ändert sich mit der theoretischen Einordnung von Emotionen allenfalls auch auf der Ebene der Phänomenologie der Emotionen einiges? Verändern sich, mit anderen Worten, nur die Kategorien, mit denen Emotionen verortet werden, oder tangiert die historische Transformation letztlich auch unser mentales Leben?

Perler selbst gibt auf diese Frage keine Antwort. Es finden sich lediglich einige Signale, die allerdings in verschiedene Richtungen weisen. Während der Titel in seiner Doppeldeutigkeit eher auf einen Antirealismus mit Blick auf die Gefühle anspielt, legen sein systematisierender Zugriff sowie der erfrischend unbefangene Umgang mit mittelalterlichen Beispielen es nahe, dass er gleichwohl anthropologische Konstanten annimmt. Hier wäre ein klarerer Positionsbezug des Autors nicht zuletzt deshalb aufschlussreich gewesen, weil davon auch die Frage tangiert ist, was Transformation von Emotionen in einem systematischen Sinn des Wortes genau heißen kann. ${ }^{2}$

Der zweite Punkt betrifft das Verhältnis von theoretischer und praktischer Philosophie. Wie Perler explizit festhält, verbinden viele klassische Gefühlstheorien metaphysische oder naturphilosophische Annahmen mit handlungstheoretischen und moralphilosophischen Fragestellungen (vgl. vor allem 38). Das kann durchaus verallgemeinert werden: Moralpsychologische Fragen sind neben metaethischen Erwägungen typische systematische Gelenkstellen zwischen der theoretischen und der praktischen Philosophie. Hat man sich das erst einmal vergegenwärtigt, so stellt sich die Frage, ob die theoretische Reflexion auf Gefühle nicht auch

2 Vgl. für diesen Zusammenhang auch U. Renz, Philosophie als medicina mentis? Zu den Voraussetzungen und Grenzen eines umstrittenen Philosophiebegriffs, in: Deutsche Zeitschrift für Philosophie, 58 (2010), 17-30. 
ganz anders gearteten praktischen Interessen dienen kann, als das bei den genannten Autoren der Fall ist. So sind aus der Philosophie der frühen Neuzeit weitere Modelle bekannt. Beispielsweise kann die Reflexion auf Emotionen, wie das Machiavelli und Hobbes vorgeführt haben, auch im Horizont von Theorien der Herrschaft oder von Staatstheorien erfolgen. Oder eine Gefühlstheorie kann im Zuge der Auseinandersetzung mit dem Problem der moralischen Motivation entwickelt werden, wie das seit der Prägung des Begriffes des ,moral sense ' durch Shaftesbury bis in die Gegenwart hinein öfters geschah. Schaut man diese Ansätze an und vergleicht sie ihrerseits mit den von Perler vorgestellten Theorien, so wird man sehen, dass die theoretische Konzeptualisierung von Gefühlen nicht nur abhängig ist vom jeweiligen metaphysischen Rahmen, sondern auch davon, welche Themen im Bereich der praktischen Philosophie auf der Agenda eines Philosophen stehen. Das bestätigt auf der einen Seite Perlers zentrale Diagnose der historischen Bedingtheit von Gefühlstheorien, während auf der anderen Seite zugleich klar wird, dass seine Geschichte der historischen Transformation der Gefühlstheorien in der frühen Neuzeit nur eine von vielen möglichen ist.

Das Verdienst seines Buchs wird durch diese Hinweise nicht geschmälert, und wer sich auf Grund anderer systematischer Interessen oder unter anderen historischen Gesichtspunkten mit philosophischer Emotionstheorie und ihrer Geschichte befasst, wird daher von der Lektüre sehr bereichert werden. 